\title{
Imagen y reconocimiento de enfermeras ilustres a través de las monedas del mundo
}

Image and recognition of illustrious nurses through world coins

\section{Imagem e reconhecimiento de enfermeiras ilustres a través das moedas do mundo}

\author{
Juan Francisco Reyes Revuelta \\ Diplomado en Enfermería
}

Cómo citar este artículo en edición digital: Reyes-Revuelta, J.F. (2017). Imagen y reconocimiento de enfermeras ilustres a través de las monedas del mundo. Cultura de los Cuidados (Edición digital), 21(49).

Recuperado de http://dx.doi.org/10.14198/cuid.2017.49.10

Correspondencia: Equipo de Salud Mental Comunitario Puerta Blanca. Avenida Gregorio Diego, 46 CP 29004. Málaga. Correo electrónico: reyesrevuelta@hotmail.com

Recibido: 07/03/2017; Aceptado: 05/06/2017

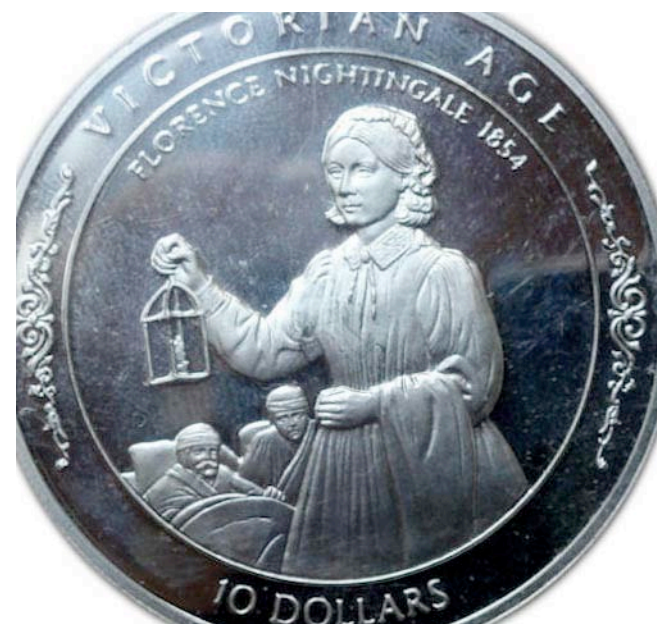

\section{ABSTRACT}

Over the last few years there has been a growing interest in iconography as an instrument for the historical research of the Nursing profession. There are still few and little known articles which use Numismatics as a source of study for the construction of the History of Care and Nursing. The present study aims, using a descriptive and documentary observation methodology, to locate and identify world coins in which are appreciated work and contributions to the profession and the society of illustrious nurses. A total of eleven coins considered relevant for the present study were selected. A small biographical account of the nurse that appears in each coin is made and coin description is made applying iconographic methodology. It is necessary to continue identifying, describing and contextualizing numismatic issues of interest for the study of the History of Care and Nursing.

Keywords: Iconography, coins, nursing history, numismatics.

\section{RESUMEN}

A lo largo de estos últimos años ha surgido un creciente interés por la iconografía, como instrumento para la investigación histórica de la profesión enfermera. Son aún escasos y poco conocidos los que utilizan la Numismática como fuente de estudio para la construcción de la Historia de los Cuidados y de la Enfermería. El presente estudio tiene como objetivo, utilizando una metodología descriptiva y de observación documental, localizar e identificar monedas mundiales donde estén reconocidas la labor y aportaciones a la profesión y a la sociedad de enfer- 
meras ilustres. Se seleccionaron un total de once monedas consideradas relevantes para el presente estudio Se realiza una pequeño relato biográfico de la enfermera que aparece en cada moneda y se realiza la descripción de las mismas aplicando metodología iconográfica. Es necesario continuar identificando, describiendo y contextualizando emisiones numismáticas de interés para el estudio de la Historia de los Cuidados y de la Enfermería.

Palabras claves: Iconografía, monedas, historia de la enfermería, numismática.

\section{RESUMO}

Nos últimos anos tem havido um interesse crescente na iconografia, como uma ferramenta para a pesquisa histórica da profissão de enfermagem. São escassos e pouco conhecidos aqueles que utilizam Numismática como fonte de estudo para a construção da História de Cuidado e Enfermagem. Este estudo visa, por meio da observação descritiva e documental, localizar e identificar moedas do mundo onde são reconhecidos o trabalho e as contribuições para a profissão e para a sociedade de enfermeiras ilustres. Um total de onze moedas consideradas relevantes foram selecionados para este estudo. Uma pequena história biográfica é feito da enfermeira que aparece em cada moeda e a descrição as mesmas é realizado através da aplicação de metodologia iconográfica. É necessário continuar a identificar, descrever e contextualizar questões numismáticas de interesse para o estudo da história de cuidados e de enfermagem.

Palavras-chave: Iconografia, moedas, história da enfermagem, numismática.

\section{INTRODUCCIÓN}

Cada vez nos encontramos con un número creciente de artículos que exploran la construcción de la Historia de la Enfermería y la Historia de los Cuidados utilizando como herramienta el análisis iconográfico. Autores como Santo Tomás $(1996,1997)$ o Núñez del Castillo $(2004,2008)$ sostienen que los signos iconográficos han de ser considerados como fuentes válidas para avanzar en el conocimiento histórico de la profesión. Siles (1999) afirma en el mismo sentido que cualquier tema, objeto o personaje relacionado con la enfermería en cualquiera de sus épocas y variantes puede ser estudiado iconográficamente. Para Siles (2011) el análisis iconográfico es una forma de análisis de contenido, que tiene identidad propia y que puede considerarse tanto como una fuente como un método de indudable valor para la Historia de la Enfermería.

Son conocidos y ampliamente difundidos trabajos de especial interés que utilizando la iconografía analizan aspectos referidos a la enfermería y la relación de ayuda y cuidado a través del cine (Siles 1994 y 2009) las emisiones filatélicas (Ballesteros, 2006; Guerra 2008; Luna y Miralles, 2008), la pintura (Calvo 1994), o los monumentos urbanos (Ávila 2014, Casas y Miralles, 2001; Miralles y Duran 2005). Sin embargo, son aún escasos y poco conocidos los que utilizan la Numismática como una original, alternativa e interesante fuente de estudio para la construcción de la Historia de la Enfermería y de los Cuidados. Esta línea de investigación se abordó inicialmente con los trabajos de J.M. Guerra y C.Ma Martínez: "Las Monedas de los Leprosarios en el Standard Catalog of World Coins 1901-1908” (2008), "La Numismática. Fuente de Investigación para la Historia de los Cuidados" (2009) e "Iconografía cuidadora en el texto numismático. Standard Catalog of World Coins 1901-2000" (2010). Encontramos en la literatura científica un interesante ensayo escrito por la profesora 
brasileña Moreira et al. "Marcas simbólicas de la historia de la Enfermería: el caso de la moneda brasileña de 400 reales de 1936" (2009). Recientemente cabe citar el artículo publicado por J.F. Reyes "Imagen de la práctica de la lactancia materna a través de la Numismática contemporánea" (2014) y la comunicación "La Enfermería en las monedas del Mundo" (2015) por M. Herrero et al. presentada en el congreso nacional e internacional de Historia de la Enfermería celebrado en el año 2015 en la ciudad de Santander.

Con el objetivo de continuar identificando, describiendo y contextualizando emisiones numismáticas con representaciones de enfermeras o fenómenos relacionados con el cuidado se presenta el siguiente artículo, que pretende catalogar y describir las monedas mundiales donde estén reconocidas la labor y aportaciones a la profesión y a la sociedad de enfermeras ilustres.

\section{METODOLOGÍA}

Para la realización del presente estudio descriptivo, de observación documental, se analizaron catálogos de monedas contemporáneas, principalmente el catálogo Krause World-Coins en castellano y el catálogo en red Colnect, utilizando como término de búsqueda en primera criba “Enfermera”. Éste primer término se conjugó con los siguientes términos: "Enfermería”, "Hospital", "Salud" y "Cuidados", en las siguientes ecuaciones de búsqueda avanzada.
En total, se recuperaron 22 monedas que se sometieron a los siguientes criterios de inclusión / exclusión.

Criterios de inclusión: Monedas de curso legal y monedas conmemorativas de cualquier país en las que aparezcan la imagen o el nombre de una enfermera ilustre, con titulación reconocida, emitidas dese el año 1900 hasta el año 2015.

Criterios de exclusión: Monedas que representan la imagen de otros personajes ilustres que si bien en algún momento de su biografía, como por ejemplo misiones, obras sociales o guerras, ejercieron de sanitarias o enfermerasprácticas, no poseen titulación reconocida, ni el motivo de emisión de la moneda fue reconocer su trayectoria o aportaciones como enfermera. También se excluyen las monedas en las que se representen la imagen de enfermeras anónimas aunque el motivo de la emisión sea

\begin{tabular}{|c|c|}
\hline ENFERMERA ILUSTRE & MONEDA (pa $s$ emisor/ valor facial/ a $\square$ omisi $\square$ n) \\
\hline Esma Deniz & Turqu $a$ 20 liras turcas 2015 \\
\hline Sophie Mannerheim & Finlandia 10 euros 2013 \\
\hline Georgina Pope & Canad $\square 5 \mathrm{~d}$ lares 2012 \\
\hline Edith Cavell & $\begin{array}{l}\text { Islas Virgenes Brit } \_ \text {nicas } 1 \mathrm{~d} \sqcap \text { lar } 2014 \\
\text { Reino Unido } 5 \text { libras } 2015\end{array}$ \\
\hline Florence Nightingale & $\begin{array}{l}\text { Islas Solomon } 10 \mathrm{~d} \text { lares } 1996 \\
\text { Gibraltar } 1 \text { corona } 2001 \\
\text { Alderney } 5 \text { libras } 2004 \\
\text { Alderney } 5 \text { libras } 2004 \\
\text { Jersey } 5 \text { libras } 2006 \\
\text { Reino Unido } 2 \text { libras } 2010\end{array}$ \\
\hline
\end{tabular}

Tabla 1. Resumen de las monedas seleccionadas para el presente estudio. 
un reconocimiento a determinada labor o servicios de enfermería.

Tras aplicar los criterios de inclusión y exclusión y completar la búsqueda de forma intuitiva a través de Google, se seleccionaron finalmente un total de once monedas consideradas relevantes para la presente revisión, su trascendencia y comprensión (ver cuadro resumen en tabla 1). Se realiza una pequeña descripción biográfica de la enfermera que aparece en cada moneda y se catalogan en base al año de emisión, país emisor, valor facial y rótulo. Por último, se realiza la descripción de las mismas aplicando metodología iconográfica.

\section{RESULTADOS}

\section{ESMA DENIZ}

\section{Breve descripción biográfica}

Nació en la ciudad turca de Kavala en 1902 y murió en 1997. Dedicó 73 años de su vida a la enfermería y murió a los 95 años de edad. Fue uno de los

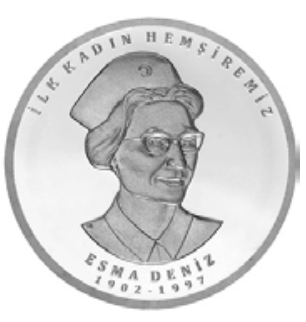
miembros fundadores de la Asociación de Enfermeras de Turquía y asumió el cargo de jefe de la asociación durante 18 años. Esma Deniz representó a las enfermeras turcas en la Unión Internacional de la Enfermera y se le dio el título de "Enfermera de Comunidad de Salud de Turquía”. Participó en la organización de la Escuela privada de Enfermería Kizilay y contribuyó a la creación de la Escuela de Enfermería de Florence Nightingale.

Año de Emisión: 2015

País emisor: Turquía

Valor facial: 20 Liras Turcas

Rótulo: ILK KADIN HEMSIREMIZ / ESMA

DENIZ/ 1902-1907

\section{Descripción iconográfica de la moneda}

El reverso de la moneda muestra el retrato de Esma Deniz con uniforme de enfermera y cofia, donde aparece una media luna. Se puede leer en la parte superior la inscripción en turco "ilk kadin hemşiremiz" que se traduce como "nuestra primera mujer enfermera". En la parte inferior aparece el rótulo del año de nacimiento y muerte de Esma Deniz.

\section{SOPHIE MANNERHEIM}

\section{Breve descripción biográfica}

Nacida en 1863 fue pionera de la enfermería moderna en su país. Se le considera una mujer adelantada a su tiempo y prototipo de mujer moderna. Fue una precursora independiente y una figura prominente de su tiempo, trabajando para desarrollar las prácticas de enfermería y promover el bienestar de los niños El trabajo de la vida de Sophie Mannerheim es una inspiración para todos los que valoran la enfermería y el cuidado. Dejó atrás la vida ociosa de una dama aristocrática y comenzó a estudiar enfermería. Esta decisión tendría un tremendo impacto en la atención sanitaria finlandesa. Durante su carrera modernizó la formación de enfermería finlandesa y también fue una figura destacada cuando se estableció la Liga Mannerheim para el Bienestar Infantil.
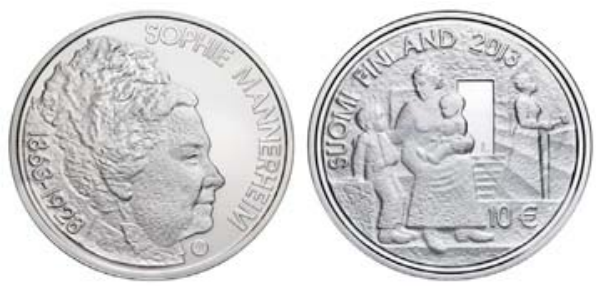

Año de Emisión: 2013

País emisor: Finlandia

Valor facial: 10 Euros

Rótulo anverso: SOPHIE MANNERHEIM /

1863-1928

Rótulo reverso: SUOMI FINLAND 2013 


\section{Descripción iconográfica de la moneda}

Moneda conmemorativa dedicada al 150 aniversario del nacimiento de la enfermera finlandesa Sophie Mannerheim. En el anverso se representa su retrato mirando hacia la derecha con la inscripción de su nombre y año de nacimiento y muerte. En el reverso se puede ver una escena de dos enfermeras trabajando en un hospital infantil tras una puerta abierta. Una de ellas, la que aparece en primer plano es Sophie Mannerheim sentada junto a un niño con brazo en cabestrillo al que mira y con bebé en su regazo, escena que representa su amor y dedicación por los niños. Los beneficios de la venta de esta moneda de colección fueron al completo dedicados a la construcción de un hospital infantil en Helsinki.

\section{GEORGINA POPE}

\section{Breve descripción biográfica}

Enfermera canadiense nacida en 1862. Después de completar los estudios de Enfermería en 1899 en el

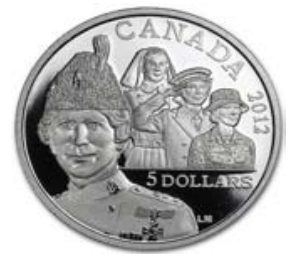
Hospital Bellevue en la ciudad de Nueva York, se ofreció como voluntaria para el servicio de enfermería en la segunda guerra Boer en Sudáfrica. Esta cita fue un momento significativo en la evolución de la participación de las mujeres en el ejército canadiense. Por primera vez, a las mujeres que actuaban como enfermeras se les dio un rango militar, así como el salario atribuido a ese rango. Debido a Georgina Pope, las Fuerzas Canadienses de hoy, incluyendo todas las ramas del Servicio están abiertas a las mujeres. Tras finalizar la guerra en Sudáfrica, en 1902 regresó como comandante del Servicio de Enfermería del Ejercito Canadiense. En 1903 se convirtió en la primera mujer canadiense en recibir la
Cruz Roja Real, que se le otorgó por su servicio meritorio y distinguido en el campo de batalla. En 1917, a los 55 años, Georgina Pope, a pesar de su mala salud, fue a trabajar cerca de la ciudad holandesa de Ypres donde sirvió durante el resto de la I Guerra Mundial hasta 1918.

\section{Año de Emisión: 2012 \\ País emisor: Canadá \\ Valor facial: 5 dólares}

\section{Descripción iconográfica de la moneda}

En la moneda se representan cuatro retratos. En primer plano Georgina Pope con uniforme militar canadiense y portando la medalla de la Cruz Roja Real. En el fondo aparecen tres mujeres militares del siglo pasado, incluyendo una enfermera de la Primera Guerra Mundial, un miembro del Servicio naval Real de las Mujeres de la Segunda Guerra Mundial y una mujer miembro del ejército canadiense de hoy.

\section{EDITH CAVELL}

\section{Breve descripción biográfica:}

Fue una enfermera británica nacida en 1865 y condenada a muerte en 1915 en juicio sumario por un tribunal militar alemán durante la

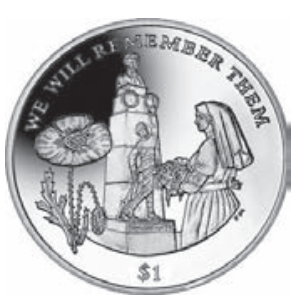
Primera Guerra Mundial, por haber cobijado en su hospital en Bruselas hasta doscientos soldados belgas, franceses e ingleses (prisioneros evadidos y pilotos abatidos) y haberles ayudado a huir de Bélgica y reintegrarse a sus puestos de combate. La noticia de su sentencia a muerte asombró y enfureció a los países aliados, así como a varios neutrales como Estados Unidos o España, que solicitaron sin éxito que le fuese respetada su vida. 
Año de Emisión: 2014

País emisor: Islas Vírgenes Británicas

Valor facial: 1 dólar de Islas Vírgenes

Rótulo: WE WILL REMEMBER THEM

\section{Descripción iconográfica de la moneda}

Moneda incluida dentro de una serie de monedas que las Islas Vírgenes Británicas emitieron durante ese año con el lema "WE WILL REMEMBER THEM", "LES RECORDAREMOS" traducido al castellano, dedicada a conmemorar hechos y personajes significativos de la Primera Guerra Mundial. En la moneda se representa el monumento-monolito que la ciudad inglesa de Norwich dedicó a su heroína y mártir Edith Cavell. A sus pies una enfermera anónima deposita un ramo de flores como gesto de reconocimiento. En la parte izquierda de la moneda aparece representada la flor de la amapola como símbolo de los soldados caídos durante la Primera Guerra Mundial. La costumbre de relacionar las amapolas con la guerra viene de la época napoleónica cuando un escritor se percató que los campos de batallas tras un conflicto se cubrían de estas flores en primavera.

Año de Emisión: 2015

País emisor: Reino Unido

Valor facial: 5 Libras

Rótulo: 1914 THE FIRST WORLD WAR 1918 / EDITH CAVELL

Inscripción en el canto: THEY FACED THEM GENTLE AND BOLD

\section{Descripción iconográfica de la moneda}

Esta moneda también aparece incluida dentro de otra serie de seis monedas que el Reino Unido emitió para conmemorar el centenario de la Primera Guerra Mundial. En primer plano y en la parte inferior derecha se representa una imagen de Edith Cavell atendiendo a un soldado herido, pero también mirando por encima del hombro, vigilante y valiente, para asegurarse de que no hay peligro y ayudar al soldado herido a escapar. En segundo plano se ha representado un retrato de Edith Cavell con cofia. Alrededor de la moneda nuevamente aparecen amapolas como símbolo de los caídos en la Primera Guerra Mundial y espinas como símbolo de su detención y ejecución. En la inscripción del canto de la moneda se lee "SHE FACED THEM GENTLE AND BOLD" inspiradas por las palabras que el poeta Laurence Binvon dedicó a Edith Cavell, y que se traducen como "ELLA SE ENFRETÓ A ELLOS DE FORMA SERENA Y VALIENTE”.

\section{FLORENCE NIGHTINGALE}

\section{Breve descripción biográfica:}

Enfermera inglesa nacida en la ciudad italiana de Florencia en 1820 y fallecida en 1910. Es considerada una de las pioneras en la práctica de la

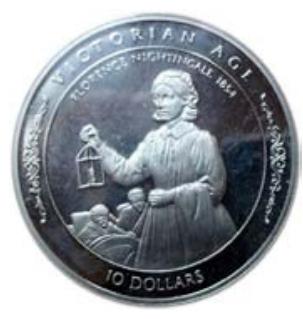
enfermería y creadora del primer modelo conceptual de enfermería. Proveniente de la case alta, se rebeló contra los prejuicios de su época y contra su destino de mujer que debía permanecer en el hogar y, ante la oposición de sus padres, escogió la profesión de enfermera. En 1853, llegó a ser supervisora de enfermeras de un hospital de caridad de Londres, en el que introdujo grandes innovaciones técnicas y de organización, pero su fama se incrementó organizando un servicio de enfermeras para los soldados británicos en la Guerra de Crimea, donde consiguió me- 
joras sanitarias espectaculares. Desplegó una actividad frenética, consiguió la reforma de la Sanidad militar británica, la extensión progresiva de su modelo a la sanidad civil, la introducción de reformas sanitarias en la India y la creación de una escuela de enfermeras.

Año de Emisión: 1996

País emisor: Islas Solomon

Valor facial: 10 dólares de Islas Solomon

Rótulo: VICTORIAN AGE / FLORENCE NIGHTINGALE 1844

\section{Descripición iconográfica de la moneda}

Se representa la imagen de Florence Nightingale entre soldados enfermos y heridos durante la guerra de Crimea

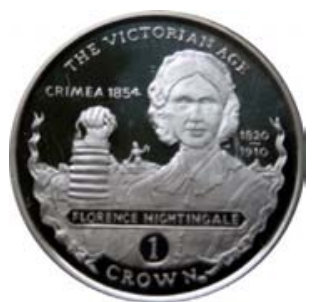
sosteniendo una lámpara en la mano. En dicha guerra tomó el apodo o sobrenombre de "La Dama de la Lámpara" ya que, por las noches, cuando todo el equipo de enfermeras y médicos se habían retirado, ella hacía interminables rondas en solitario para observar el estado de los pacientes y velar por ellos.

Año de Emisión: 2001

País emisor: Gibraltar

Valor facial: 1 Corona

Rótulo: THE VICTORIAN AGE / CRIMEA 1854 / 1920-1910/ FLORENCE NIGHTINGALE

\section{Descripición iconográfica de la moneda}

Moneda conmemorativa de la época victoriana, época que se extendió durante el largo reinado de la monarca británica Victoria I, en la que podemos observar en primer plano el retrato de Florence Nightingale sosteniendo una lámpara, en alusión a su conocido apodo, con una versión más pequeña de ella atendiendo un

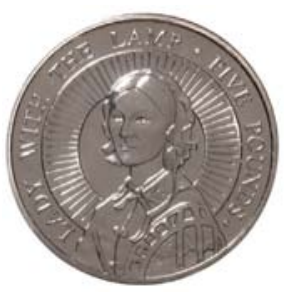
paciente en el fondo.

Se inscribe el nombre y la fecha de nacimiento y muerte de Florence Nightingale y se inscribe además "CRIMEA 1854", hito destacado de la época victoriana.

Año de Emisión: 2004

País emisor: Alderney

Valor facial: 5 libras esterlinas

Rótulo: LADY WITH THE LAMP

\section{Descripición iconográfica de la moneda}

Hermosa pieza en la que se representa el retrato de Florence Nightingale mirando hacia la izquierda en la que se inscribe el rótulo "LADY WITH

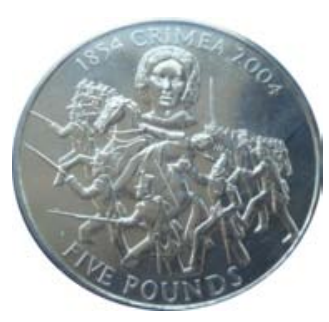
THE LAMP”, traducida como "DAMA DE LA LÁMPARA” que como hemos referido anteriormente, era el apodo con la que se conoció a Florence Nightingale partir de la guerra de Crimea.

Año de Emisión: 2004

País emisor: Alderney

Valor facial: 5 libras esterlinas

Rótulo: 1854 CRIMEA 2004

\section{Descripición iconográfica de la moneda}

Esta moneda se incluye dentro de un set de tres monedas y una medalla emitidas por la isla del Canal de Alderney para conmemorar 
el 150 aniversario de la guerra de Crimea. Se representa el busto de Florence Nightingale sobre una escena de carga de la batalla de Inkermán. La ba-

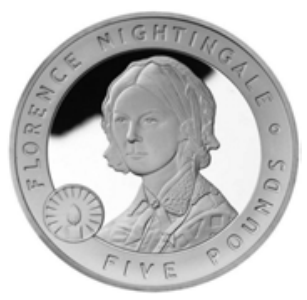
talla de Inkermán se libró el 5 de noviembre de 1854 en Crimea, y concluyó con la victoria de los ejércitos británico y francés contra el ejército ruso. Existen dos versiones de esta moneda, en una de ellas con menor volumen de emisión, donde uno de los soldados que aparece en la moneda está coloreado.

Año de Emisión: 2006

País emisor: Jersey

Valor facial: 5 libras esterlinas

Rótulo: FLORENCE NIGHTINGALE

\section{Descripición iconográfica de la moneda}

Esta onza de plata es parte de una serie de monedas emitidas por la Isla de Jersey para conmemorar personajes británicos ilustres. Se inscribe su

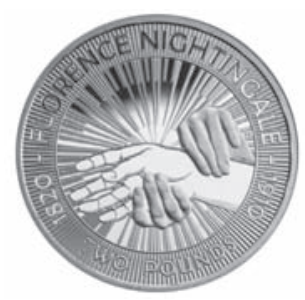
nombre y se represen-

ta nuevamente, además del busto de Florence Nightingale, la imagen de una lámpara, en la parte inferior izquierda, en alusión al apodo con la que fue conocida en todo el mundo, "La Dama de la Lámpara”.

Año de Emisión: 2010

País emisor: Reino Unido

Valor facial: 2 libras esterlinas

Rótulo: 1820 FLORENCE NIGHTINGALE 1910 Inscripción en el canto: 150 YEARS OF NURSING

\section{Descripición iconográfica de la moneda}

Se trata de una moneda bimetálica de gran belleza y con un destacado volumen de emisión que Reino Unido emitió en 2010 con motivo del $100^{\circ}$ aniversario de la muerte de Florence Nightingale. La moneda muestra las manos de una enfermera tomando el pulso bajo los rayos de luz de una lámpara. La elección de las manos como imagen representativa de la función enfermera se adecua perfectamente al mensaje que se pretende expresar. Las manos acarician, saludan, curan, lavan, dan, toman, sostienen, sujetan... En el rótulo se puede leer "1820-FLORENCE NIGHTINGALE-1910” y en la inscripción del canto “150 YEARS OF NURSING" ya que esta moneda conmemora además el 150 ${ }^{\circ}$ aniversario de la publicación de su libro "Notas de Enfermería: qué es y qué no es", donde se recogen las conclusiones de más de catorce años de observación, experiencia y pensamiento de la autora durante el ejercicio de su profesión.

\section{CONCLUSIONES}

Se han identificado, catalogado y analizado iconográficamente once monedas dedicadas a cinco enfermeras ilustres, con nombre propio, de nueve países diferentes.

Gracias a que las monedas se comportan como magníficos elementos de propaganda y de difusión y debido además al hecho de que se consideran valiosas entre la población, las piezas presentadas en el presente estudio se convierten en un homenaje público de gratitud y consideración, de primer orden, por parte aquellos países que emitieron estas monedas, reconociendo no únicamente las aportaciones y labor individual de las enfermeras representadas, sino también a la totalidad de la profesión de Enfermería en general. 
Las monedas se comportan como una válida y original fuente alternativa de estudio, aún por desarrollar, para la construcción de la Historia de los Cuidados y de la Enfermería. Se hace por tanto necesario seguir explorando nuevas líneas de investigación en este sentido y continuar identificando, describiendo y contextualizando emisiones numismáticas con representaciones de enfermeras o fenómenos relacionados con el cuidado.

\section{BIBLIOGRAFÍA}

- Ávila, J.A. (2014). Los signos iconográficos del reconocimiento público de la Enfermería en España: su importancia y significación. Cultura de los Cuidados (Edición digital) 18, 38. Disponible en http://dx.doi.org/10.7184/ cuid.2014.38.12> consultado el 12 de diciembre de 2016

- Ballesteros, A.M. (2006). La Enfermería española y su historia en la filatelia. Temperamentum, 4. Disponible en http://indexf.com/temperamentum/tn4/t6245.php> consultado el 14 de diciembre de 20016.

- Calvo, M.A. (199). Análisis iconográfico de la pintura de Lucas Valdés "Asistencia a los sacerdotes en la enfermería”. Híades, 10, 403-412

- Casas, M. F., y Miralles, M.T. (2001). Monumento a las enfermeras de la Cruz roja de Astorga. Enfermería clínica, (4), 161-165

- Guerra, J.E. (2008).Historia de la enfermería. Reflejo de la Filatelia. Híades, 10, 782-803.

- Guerra, J.E., y Martínez , C.M. (2008). Las monedas en los Leprosarios en el Standard Catalog of World Coins 1901-1998. X Congreso Nacional y V Internacional de Hisotria de la Enfermería, Almería.

- Guerra, J.E., y Martínez, C.M. (2009). La Numismática. Fuente de Investigación para la Historia de los Cuidados. Temperamentvm ,10. Disponible en http://www.indexf. com/temperamentum/tn10/t7227.php > Consultado el 8 de diciembre de 2016.

- Guerra, J.E., y Martinez, C.M. (2010). Iconografía cuidadora en el texto numismático "Standard Catalog of Word Coins 1901-2000”. Hiádes, 11, 539-554.

- Herrero, M., Parrás, P., y Olavarría, E. (2015). La Enfermería en las monedas del mundo. XIV Congreso Nacional y IX Internacional de Historia de la Enfermería. Santander, 7 al 9 de mayo.

- Luna, S. (2004). La Enfermería en la filatelia. Híades, 9, 465-499.

- Miralles, M. T. (2008). La Enfermería a través de los sellos. Madrid:Ediciones DAE.

- Miralles, M.T., y Duran, M. (2005). La enfermera en el monumento madrileño (1908-1936). Index de Enfermería, 1, 65-69
- Moreira, A., Porto, F., Oguisso, T., y de Souza Campos, P.F. (2009). Marcas simbólicas de la historia de la enfermería: el caso de la moneda brasileña de 400 reales (1936). Enfermería Global 16, 1-11.

- Núñez, M., y Siles, J. (2004). Evolución de los cuidados enfermeros. Análisis iconográfico desde la perspectiva de Virginia Henderson. Cultura de los cuidados, 8(15), 17-25.

- Núñez, M., Siles, J., y Jurado, I. (2008). Relación de ayuda a través de la historia: una aportación desde la perspectiva iconográfica. Cultura de los Cuidados, 12 (23), 27-34

- Reyes, J.F. (2015). Imagen de la lactancia materna a través de la numismática contemporánea. Cultura de los Cuidados (Edición digital) 19, 41. Disponible en http:// dx.doi.org/10.14198/cuid.2015.41.10> consultado el 19 de diciembre de 2016.

- Santo Tomás, M., et al. (1996). Iconografía y enfermería: un instrumento para la investigación histórica. Index de enfermería, (16-17), 87-88.

- Santo Tomás M. et al. (1997). Iconografía y Enfermería. Un instrumento para la investigación histórica. Index de Enfermería, 19, 13-16.

- Siles, J. et al. (1994). La enfermería a través del cine: un estudio histórico y transcultural. Híades, 10, 761- 780.

- Siles, J. (1999). Historia de la Enfermería. Alicante: Aguaclara.

- Siles, J. (2009). Origen de la Enfermería en el cine: El género histórico-documental y bibliográfico. Cuadernos de Cine,4, 57-69.

- Siles, J. (2011). Historia de la Enfermería.Madrid: Ediciones DAE. 